\title{
Power Vector Generation Tool for Free-Space Optical Links - PVGeT
}

\author{
Dirk Giggenbach \\ Swaminathan Parthasarathy \\ Amita Shrestha \\ Florian Moll \\ Ramon Mata-Calvo
}

\author{
Institute of Communications and Navigation (IKN) \\ German Aerospace Center (DLR) \\ 82234 Wessling, Germany
}

\begin{abstract}
Reliable modelling of the power variations in atmospherically propagated optical waves is important for appropriate design of the necessary fading mitigation techniques like Forward Error Correction (FEC) or Automatic Repeat reQuest (ARQ) algorithms. A simulation tool modeling the combined impact of atmospheric turbulence and pointing jitter is presented and its performance is compared with measured power vectors.
\end{abstract}

Keywords- FSO, channel simulation, fading, scintillation, pointing jitter, beam wander, artificial power vector, $P V G e T$

\section{INTRODUCTION}

The stability of Free Space Optical Communication Links (FSO) suffers from atmospheric index-of-refraction turbulence (IRT), and pointing errors of the transmitter telescope. The combination of both effects results in intensity scintillation and fading at the receiver telescope and corresponding signal variations at the receiver front-end. Reliable modelling of the power variations is important for appropriate design of the necessary fading mitigation techniques like Forward Error Correction (FEC) or Automatic Repeat reQuest (ARQ) algorithms.

Direct experimental testing of new algorithms and implementations is rather inefficient in terms of effort and cost. Especially the optimization of error-control mechanisms requires very long received signal vectors with controlled statistical behavior. Another reason for an artificial power vector generation can be that only statistical parameters describing the vector are available, but not the vector itself. Therefore, numerical simulations with received power vectors over time - depending on several parameters like aperture diameter, beam divergence, pointing-jitter, strength of atmospheric scintillations, orthogonal velocity of wind and platform movements - is required.

We present a simulation tool that models the combined impact of atmospheric turbulence and pointing jitter, a MATLABbased simulation program called PVGeT (Power Vector Generation Tool). The focus is on the simple introduction of measured or analytically derived parameters like Power Scintillation Index (PSI) and bandwidth. Normally distributed seed random variables are scaled spectrally according to theory [1] or measurement and then nonlinear functions are applied, which finally produces the lognormal and betadistributed time series of received power.

Real-world parameter sets are introduced into the simulation environment and the resulting vectors are compared to measurement results. The modelling yields long power vectors for any atmospherically impacted link scenario, e.g. optical satellite-downlinks and -uplinks [2], aircraft-downlinks [3], ground-to-ground [4], and optical links between stratospheric HAPs (High-Altitude Platforms) [5].

The primary application is currently for testing fading mitigation techniques in satellite-ground links. Also, the generated vectors are used to drive a laboratory fading testbed that emulates power fading [6].

\section{Method of the Simulation Algorithm}

Gujar [7] described a method for the generation of random signals with specific probability density and power density spectra that was used for various purposes [8] including optical propagation under IRT [9][10]. The general idea is to use a Gaussian random number generator, do required spectral shaping, and apply a non-linear function to obtain the desired skewness. This idea is picked up and further elaborated for PVGeT

The flow diagram of PVGeT is depicted in Fig. 1. The processing comprises independent random variables for lognormal scintillation and beta-distributed pointing-jitter fading, which are multiplied for combination 


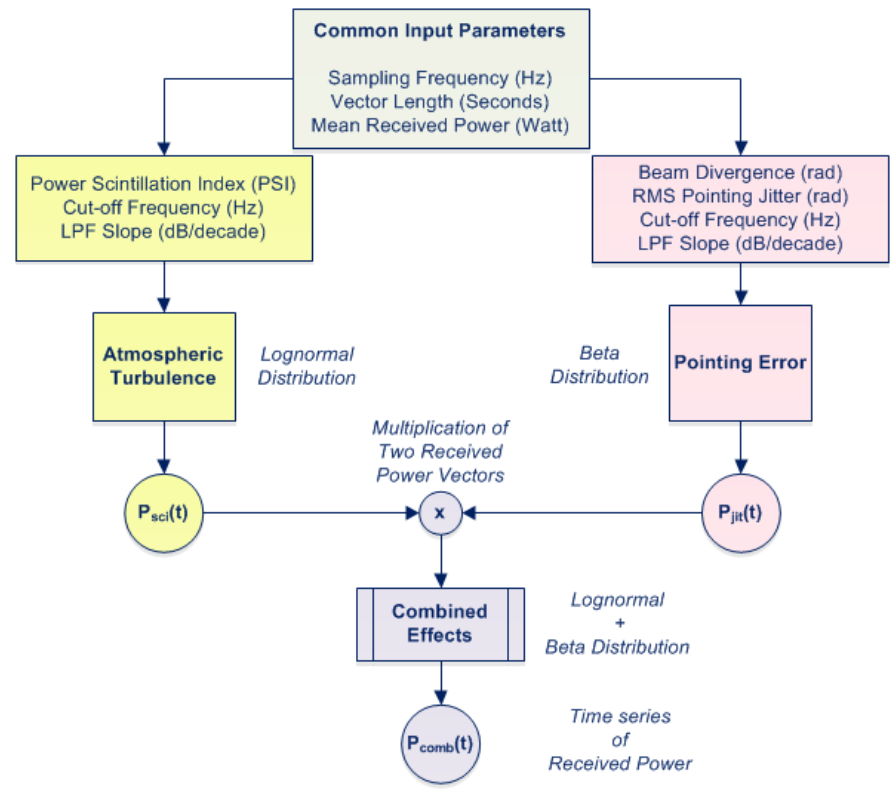

Fig. 1. PVGeT Flow Diagram

The algorithm of lognormal vector generation is illustrated in Fig. 2 and explained as follows: normally distributed random values $X_{1}, \ldots, n$ with zero mean $(\mu)$ and standard deviation $(\sigma)$ are generated and filtered using a butterworth low pass filter with cut-off frequency $\left(f_{c}\right)$ and given slope. The resulting variable $X_{1}(t)$ is scaled to the mean $(\mu)$ and standard deviation $(\sigma)$ (as derived from the targeted Power Scintillation Index). The resulting vector is the lognormally distributed received power vector $P_{s c i}(t)$.

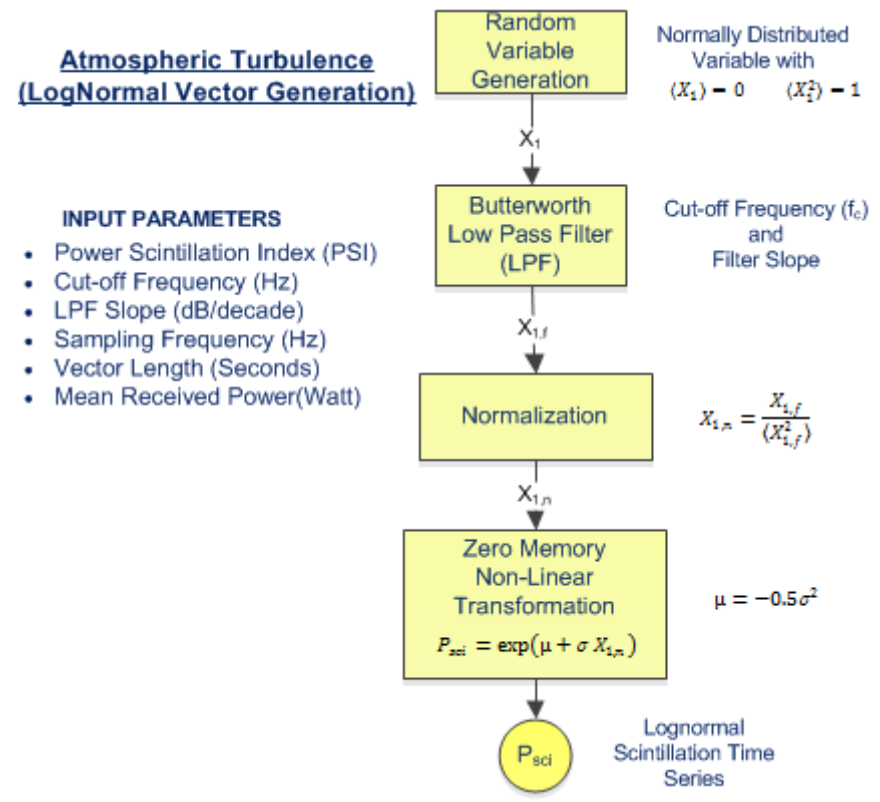

Fig. 2. PvGeT - Lognormal vector generation principle.

The pointing jitter fading vector generation is illustrated in Fig. 3. We here assume a Gaussian beam shape at the receiver and Gaussian distributed angular jitter in both orthogonal beam angles, resulting in the one-parameter type of beta distribution. First, two independent Gaussian random variables $X_{1, \ldots, n}$ and
$X_{2}, \ldots, n$ are generated with zero mean $(\mu)$ and same standard deviation one (assuming isotropic pointing error in all angular directions). $X_{1}$ and $X_{2}$ represent the pointing jitter angles in both $\mathrm{x}$ and $\mathrm{y}$ axis. Then the butterworth low-pass filters with cut-off frequency $\left(f_{c}\right)$ and slope are applied. The resulting variables are re-normalized and then the conversion from orthogonalangular to radial Rayleigh-distribution is applied to generate the radial-angular beam wander error, using the standard deviation $(\sigma)$ as calculated from the input RMS pointing jitter. Finally the fading due to the Gaussian beam shape is applied with the radial pointing error, resulting in the received power vector $P_{j i t}(t)$, fading due to pointing-errors.

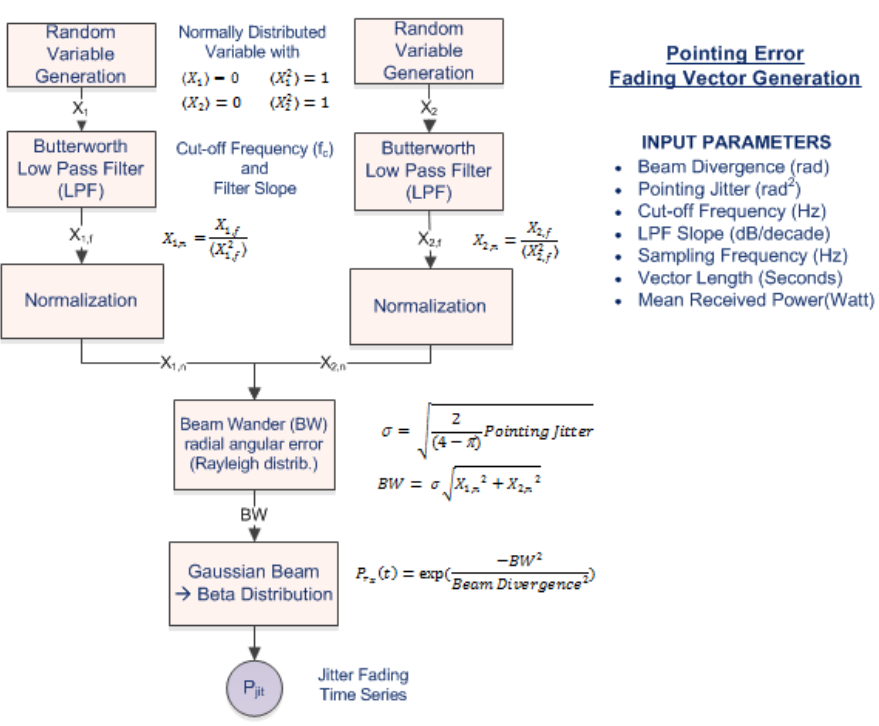

Fig. 3. PvGeT - Beta distribution vector generation principle.

\section{Comparing Generated With MEAsured Time VeCtors}

We apply PVGeT to three different transmission scenarios.

\section{A. LEO Satellite Downlink}

Recorded measurements of an optical low Earth orbit downlink (OLEODL) are used as a reference for parameterization of PVGeT. Satellite laser source was the LUCE terminal on the OICETS satellite. Receiver station was the Optical ground station in Oberpfaffenhofen $(40 \mathrm{~cm})$. Measurement wavelength was $847 \mathrm{~nm}$. The measurements are described in detail in [11]. The specific satellite pass which is used here is KT09-05, i.e. pass \#5 out of the KIODO campaign in 2009. The times series is selected at $10^{\circ}$ elevation with power scintillation index of 0.15 and auto-covariance time of $1.2 \mathrm{~ms}$ (50\% roll-off). The original and synthetic time series are shown in Fig. 4 (left: synthetic, right: measured). The length in both cases is $1 \mathrm{~s}$, power is normalized to unity mean. A signal fluctuation with a dynamic of about factor 5 is observed in both time series. 

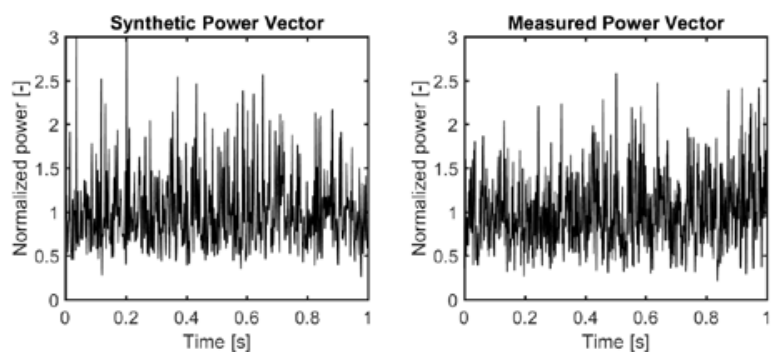

Fig. 4. OLEODL: Power-vector over time.

The corresponding probability density functions are given in Fig. 5. The black solid line denotes the PDF of the measurement, the blue solid line the synthetic vector and the red dashed line a model calculation for lognormal signal statistics. All curves match reasonably well.

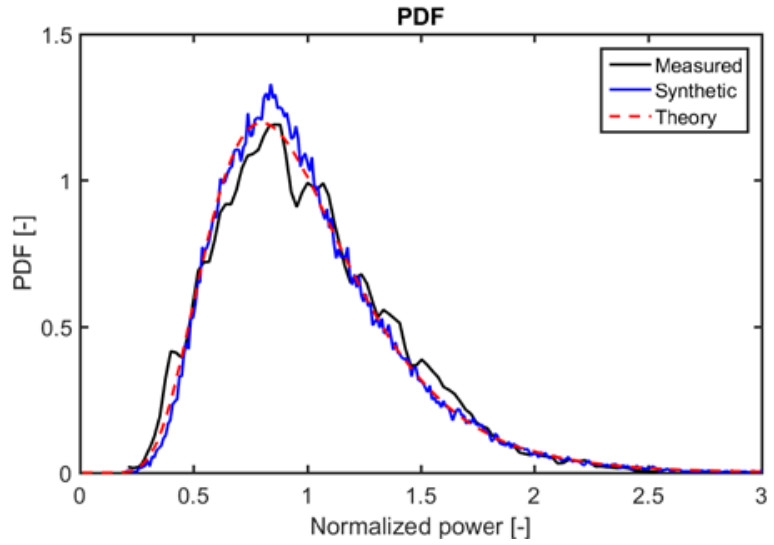

Fig. 5. OLEODL: simulated PDF vs. measured PDF

The normalized auto-covariance function of the OLEODL is shown in Fig. 6. The solid black line denotes the measurements, the dashed black line the synthetic vector. The correlation times (50\% roll-off) of the measured and the synthetic time series match reasonably well. Also, the shape of the curves matches well up to the correlation time, beyond the synthesized vector shows stronger decay.

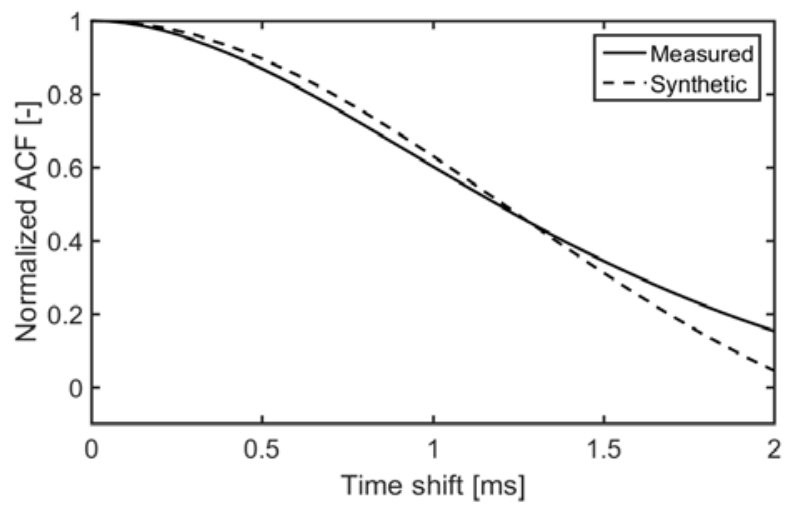

Fig. 6. OLEODL: ACOV-functions simulated and measured

\section{B. Long-Range Horizontal Link}

A long-range horizontal (LRH) link experiment over $63 \mathrm{~km}$ was conducted at an average link altitude of $150 \mathrm{~m}$ above ground using 1590nm sources and spherical beams (500 $\mu$ rad divergence). Several sample vectors were recorded in summer time, at different times before and after sunset, resulting in various IRT conditions. The one analyzed here was taken on 26. June 2016 at 18:57 local summer time, which was approx. $2.5 \mathrm{~h}$ before sunset.

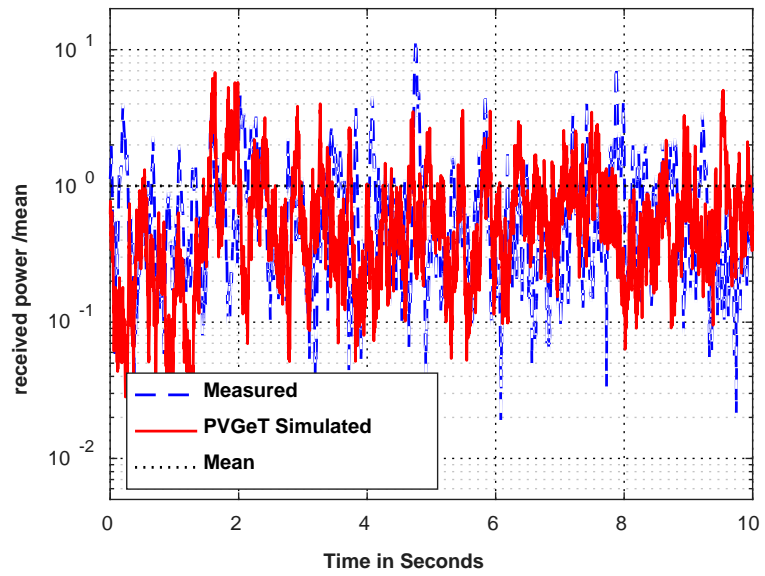

Fig. 7. LRH-scenario: Power-vector over time.

Scintillation was in the strong turbulence regime with a measured PSI of 1.87. Fig. 7 shows a comparison of normalized measured and simulated vectors, and Fig. 8 compares both PDFs. PSI of the generated vector is 1.72 .

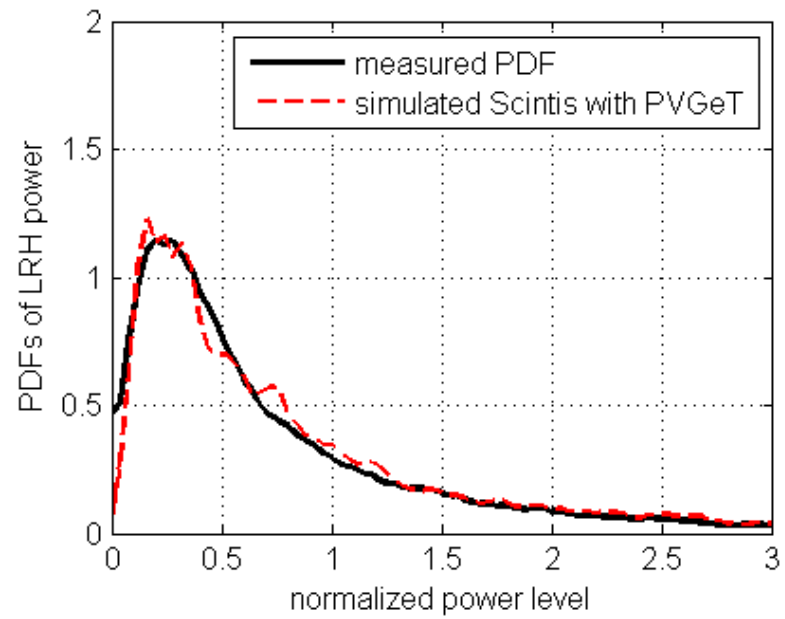

Fig. 8. LRH-scenario: simulated vs. measured PDF.

The auto-covariance functions in Fig. 9 and the scintillation bandwidth from Fig. 10 characterize the temporal behavior. 


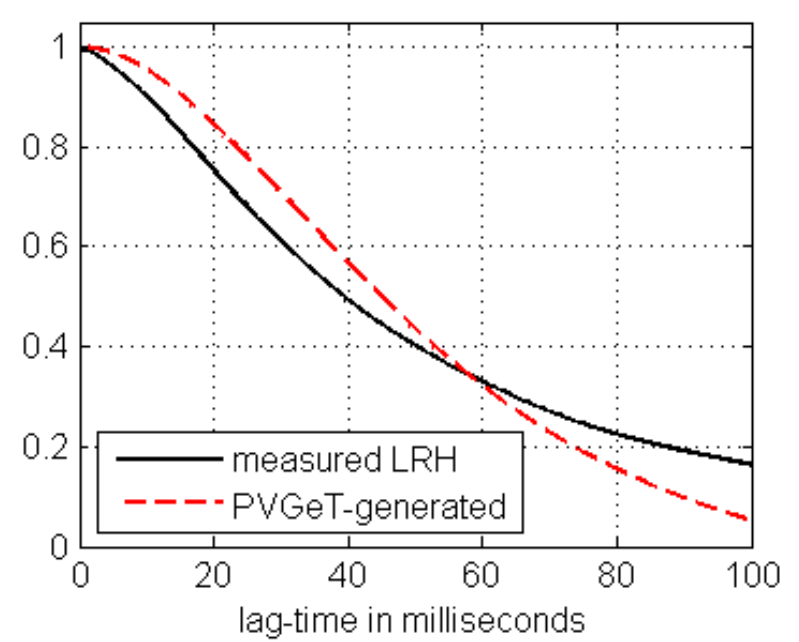

Fig. 9. LRH-scenario: ACOV-functions simulated and measured.

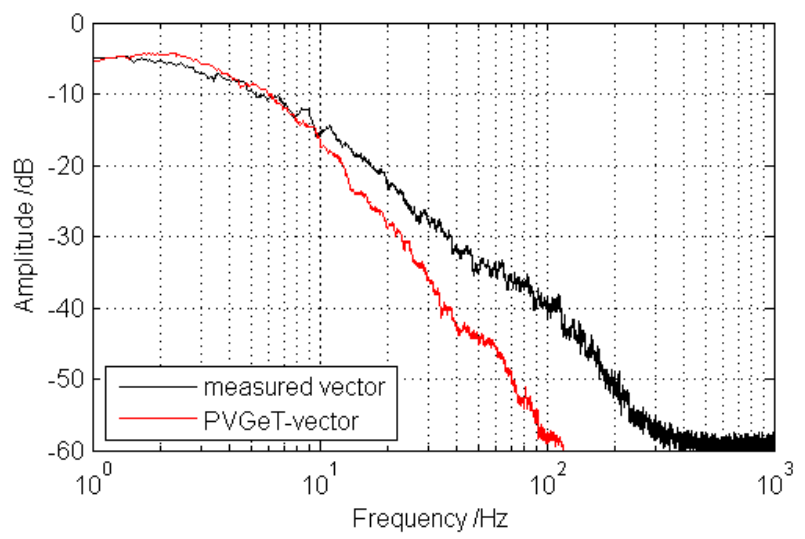

Fig. 10. Comparison of measured and PVGeT simulated power spectral density.

A rough estimation of the target scintillation bandwidth in a LRH-link simulation can be derived from the Fresnel frequency based on the mean orthogonal wind speed, as described on page 283 of [1].

\section{Impact of Pointing Jitter - Satellite Uplink}

While the scenarios for the measured vectors above are assumed to have a signal divergence much larger than the pointing jitter - or a tracking stage that corrects jitter sufficiently - in this section, we show PVGeT's ability to generate both fading effects simultaneously, by multiplying the LN-distributed scintillation vector with the beta-distributed pointing fading vector:

When the transmitted beam profile has a Gaussian shape, a parameter $\beta_{\text {jit }}$ can be defined as follows [12][13]:

$$
\beta_{j i t}=\frac{\omega_{0}{ }^{2}}{4 \cdot{\sigma_{j i t}}^{2}}
$$

with the beam intensity divergence $e^{-2}$-half-angle $\omega_{0}$ and the one dimensional beam wander sigma $\sigma_{j i t}$.

As reference measurements, we use those obtained in the ArtemEx project (experimental links between ESA-OGS at Izana on Tenerife and the geostationary satellite Artemis at 847nm signal wavelength) [14]. Fig. 12 shows a typical uplink time vector at night, for a transmit beam size of $210 \mathrm{~mm}$ (implying high beam wander), with a single uplink beam (no transmitter diversity). A PSI of 0.33 was measured for this vector.

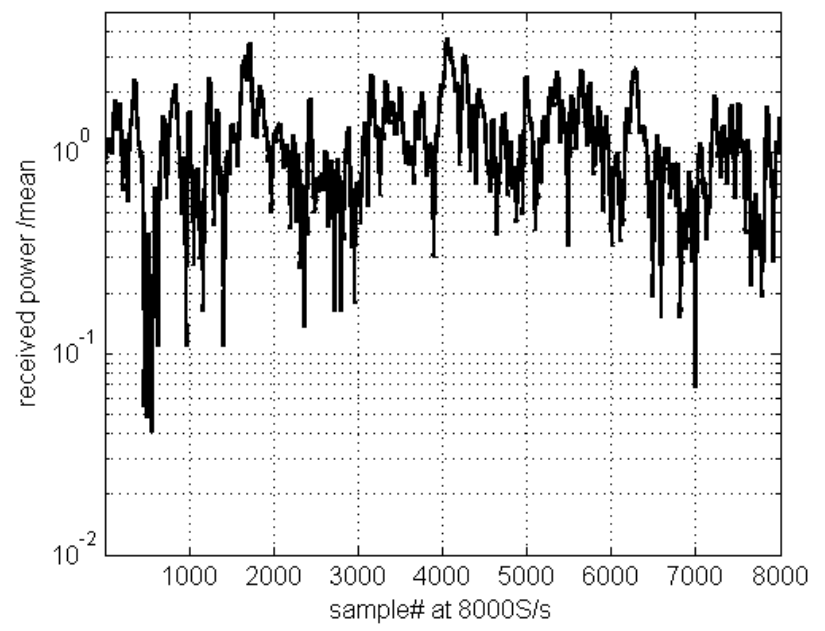

Fig. 11. Typical 1s of normalized measured uplink power variations with the 210mm diameter Tx-beam in ArtemEx, showing the strong but more infrequent fades by misspointing, while the IRT-scintillation causes equal amount of fadeand surge-level (in $\mathrm{dB}$ ) symmetric around mean.

To simulate numerically this uplink with similar statistical behavior, PVGeT was invoked with scintillation-PSI=0.18, a scintillation-bandwidth of $15 \mathrm{~Hz}$ with slope $20 \mathrm{~dB} /$ decade, pointing-jitter parameter $\beta_{j i t}=2.16$ and jitter-bandwidth of $26 \mathrm{~Hz}$ with slope $16 \mathrm{~dB} /$ decade. The resulting overall PSI then was 0.34 .

According time vectors of received power and a comparison of the measured and simulated PDF, auto-covariance functions, and spectra are shown in Fig. 12 to Fig. 15. 


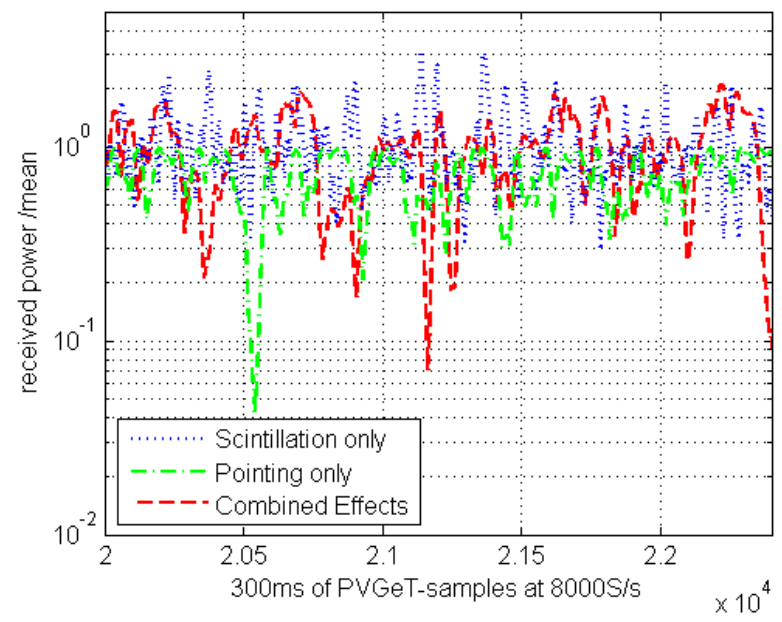

Fig. 12. Exemplary power vectors from PVGeT in one plot (300ms): scintillation-only with PSI=0.18 (blue), pointing-jitter-only with PSI=0.14 (green), and both effects combined with PSI=0.34 (red).

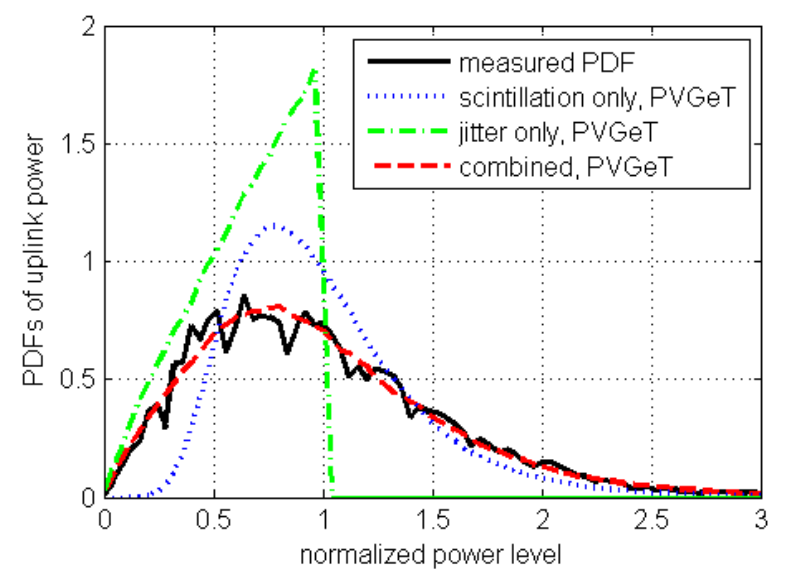

Fig. 13.PDFs of measured ArtemEx-Uplink and their simulated vector derived from the separate vectors for scintillation and pointing jitter.

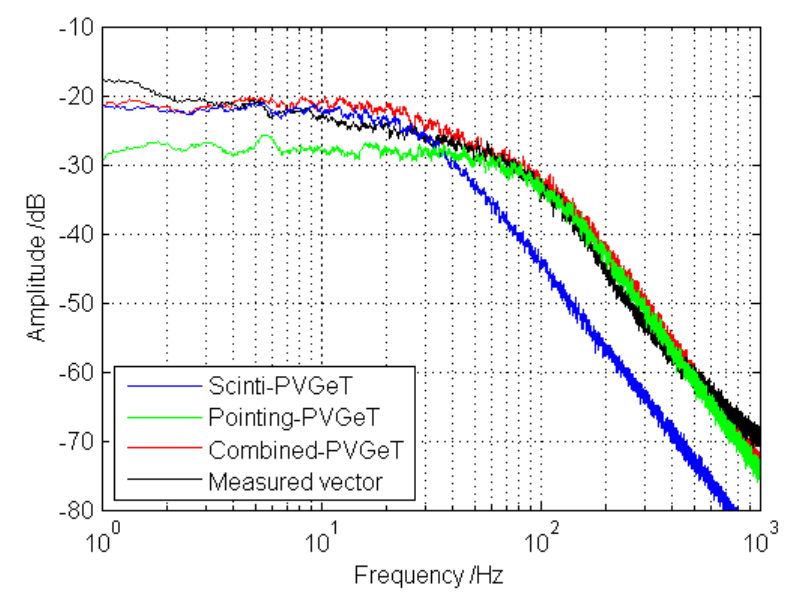

Fig. 14.Spectra of measured (black) and simulated vectors. Combined (red) is scintillation multiplied with pointing-jitter vector.

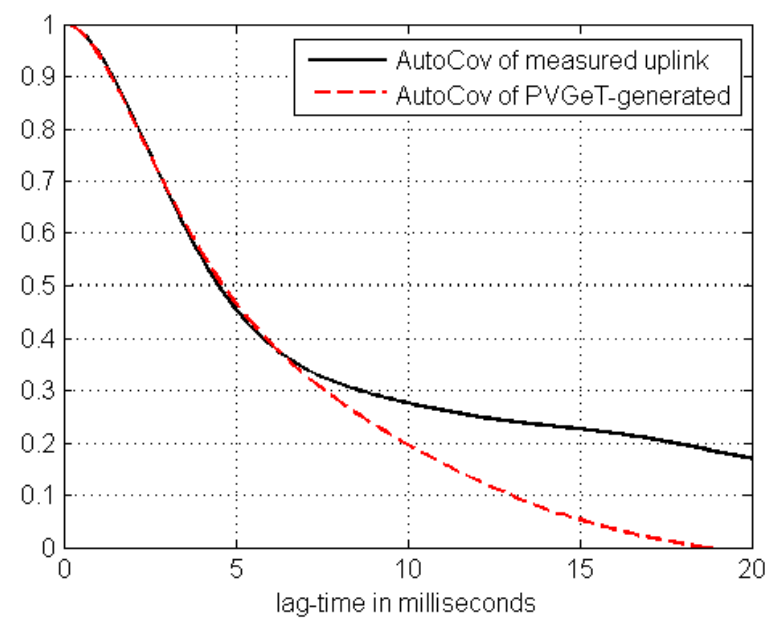

Fig. 15.Autocovariance of the GEO-uplink fading scenario.

The following table summarizes the achieved parameter values of measurements and simulations.

TABLE I. COMPARING MEASUREd AND Simulated PARAMETERS

\begin{tabular}{|l|c|c|c|}
\hline \multirow{2}{*}{} & \multicolumn{3}{|c|}{ Scenario } \\
\cline { 2 - 4 } & KIODO09-05 & Long-Range & GEO-Uplink \\
\hline $\begin{array}{l}\text { Sampling frequ. } \\
\text { measured / PVGeT }\end{array}$ & $20 \mathrm{kHz} / 10 \mathrm{kHz}$ & $10 \mathrm{kHz}$ & $8 \mathrm{kHz}$ \\
\hline PSI measured & 0.15 & 2.03 & 0.33 \\
\hline PSI from PVGeT & 0.15 & 2.01 & 0.34 \\
\hline$\beta$-param. PVGeT & - & - & 2.16 \\
\hline $\begin{array}{l}\text { PSI jitter-only } \\
\text { PVGeT }\end{array}$ & - & - & 0.14 \\
\hline Acov-HWHM-meas & $1.2 \mathrm{~ms}$ & $45 \mathrm{~ms}$ & $4.6 \mathrm{~ms}$ \\
\hline $\begin{array}{l}\text { Acov-HWHM- } \\
\text { PVGeT }\end{array}$ & $1.2 \mathrm{~ms}$ & $3 \mathrm{~Hz}$ & $25 \mathrm{~Hz}$ \\
\hline $\begin{array}{l}3 \mathrm{~dB} \text { bandwidth of } \\
\text { measured }\end{array}$ & $220 \mathrm{~Hz}$ & $2 \mathrm{~Hz}$ & $30 \mathrm{~Hz}$ \\
\hline $\begin{array}{l}3 \mathrm{~dB} \text { bandwidth of } \\
\text { PVGeT-simulated }\end{array}$ & $260 \mathrm{~Hz}$ & $-25 \mathrm{~dB} / \mathrm{dec}$. & $-41 \mathrm{~dB} / \mathrm{dec}$. \\
\hline \begin{tabular}{l} 
LP-slope-meas \\
\hline LP-slope-PVGeT
\end{tabular} & $-45 \mathrm{~dB} / \mathrm{decade}$ & $-30 \mathrm{~dB} / \mathrm{dec}$. & $-41 \mathrm{~dB} / \mathrm{dec}$. \\
\hline
\end{tabular}

\section{CONCLUSION AND OUTLOOK}

The simulation tool $P V G e T$ proofs suitable for generating long fading vectors for various scenarios, as they are necessary to investigate and optimize error-correction algorithms and other fading mitigation measures. PDFs and ACOVs measured and simulated coincide well, some ambiguity remains with the spectral slope and the tail-offset in the ACOVs. Depending on priorities one or the other can be minimized. In future, the best fitting simulation parameters may be found by iterative optimization. A final limit will be the type of distribution 
function, i.e. if lognormal- and beta-distributions are still applicable for the scenario, or if strong-turbulence distribution has to be taken into account.

The tool has been used for further scenarios not shown here, namely transmitter diversity and for simulation of intensityreciprocal channels with varying degree of correlation. A next step will be to assemble parameter sets for specific scenarios, like satellite downlinks at different elevations and apertures.

\section{REFERENCES}

[1] L. C. Andrews, R. L. Phillips, "Laser Beam Propagation through Random Media- 2nd edition”, SPIE Press, Bellingham, 2005.

[2] F. Moll, M. Knapek, "Free-space laser communications for satellite downlinks: measurements of the atmospheric channel”, In Proceedings of International Astronautical Congress (IAC 2011), October 2011.

[3] K. Shortt, D. Giggenbach, R. Mata Calvo, F. Moll, C. Fuchs, C. Schmidt, J. Horwath, J. Yeh, V. Selvaraj, R. Banerjee, "Channel characterization for air-to-ground free-space optical communication links”, Proc. SPIE 8971, 2014.

[4] D. Giggenbach, W. Cowley, K. Grant, and N. Perlot, "Experimental verification of the limits of optical channel intensity reciprocity," Appl. Opt. 51, 3145-3152, 2012.

[5] B. Moision, B. Erkmen, E. Keyes, T. Belt, O. Bowen, D. Brinkley, P. Csonka, M. Eglington, A. Kazmierski, N. Kim, J. Moody, T. Tu, W. Vermeer, "Demonstration of free-space optical communication for longrange data links between balloons on Project Loon,” Proc. SPIE 10096,
Free-Space Laser Communication and Atmospheric Propagation XXIX, February 24, 2017.

[6] A. Shrestha, D. Giggenbach, A. Mustafa, J. Pacheco, J. Ramirez, and F. Rein, "Fading testbed for free-space optical communications", Proc. SPIE 9991, Advanced Free-Space Optical Communication Techniques and Applications II, October 21, 2016.

[7] U. G. Gujar and R. J. Kavanagh, "Generation of Random Signals with Specified Probability Density Functions and Power Density Spectra," IEEE Transactions on Automatic Control, 716-719, 1968.

[8] ITU-R, P.1853-1, "Tropospheric attenuation time series synthesis", 02/2012.

[9] M. Toyoshima, H. Takenaka, Y. Takayama, "Atmospheric turbulenceinduced fading channel model for space-to-ground laser communications links”, Opt. Express 19, 2011

[10] B. Epple, "Simplified Channel Model for Simulation of Free-Space Optical Communications”, J. Opt. Comm. Netw., Vol 2, 2010

[11] "Report on DLR-JAXA Joint Experiment - The Kirari Optical Downlink to Oberpfaffenhofen (KIODO)", published by Japan Aerospace Exploration Agency JAXA, ISSN 1349-1121, Tokyo, April 2007

[12] M. Toyoshima, T. Jono, K. Nakagawa, A. Yamamoto, "Optimum divergence angle of a Gaussian beam wave in the presence of random jitter in free-space laser communication systems”, JOSA Vol. 19 No. 3, March 2002

[13] K. Kiasaleh, T.-Y. Yan, "A Statistical Model for Evaluation GOPEX Uplink Performance”, DTA progress Report 42-111. 1992

[14] R. Mata-Calvo, P. Becker, D. Giggenbach, et al, „Transmitter diversity verification on ARTEMIS geostationary satellite“, Proceedings of the SPIE 8971, Feb. 2014. 